Supplementary figures and tables for Döring et al.:
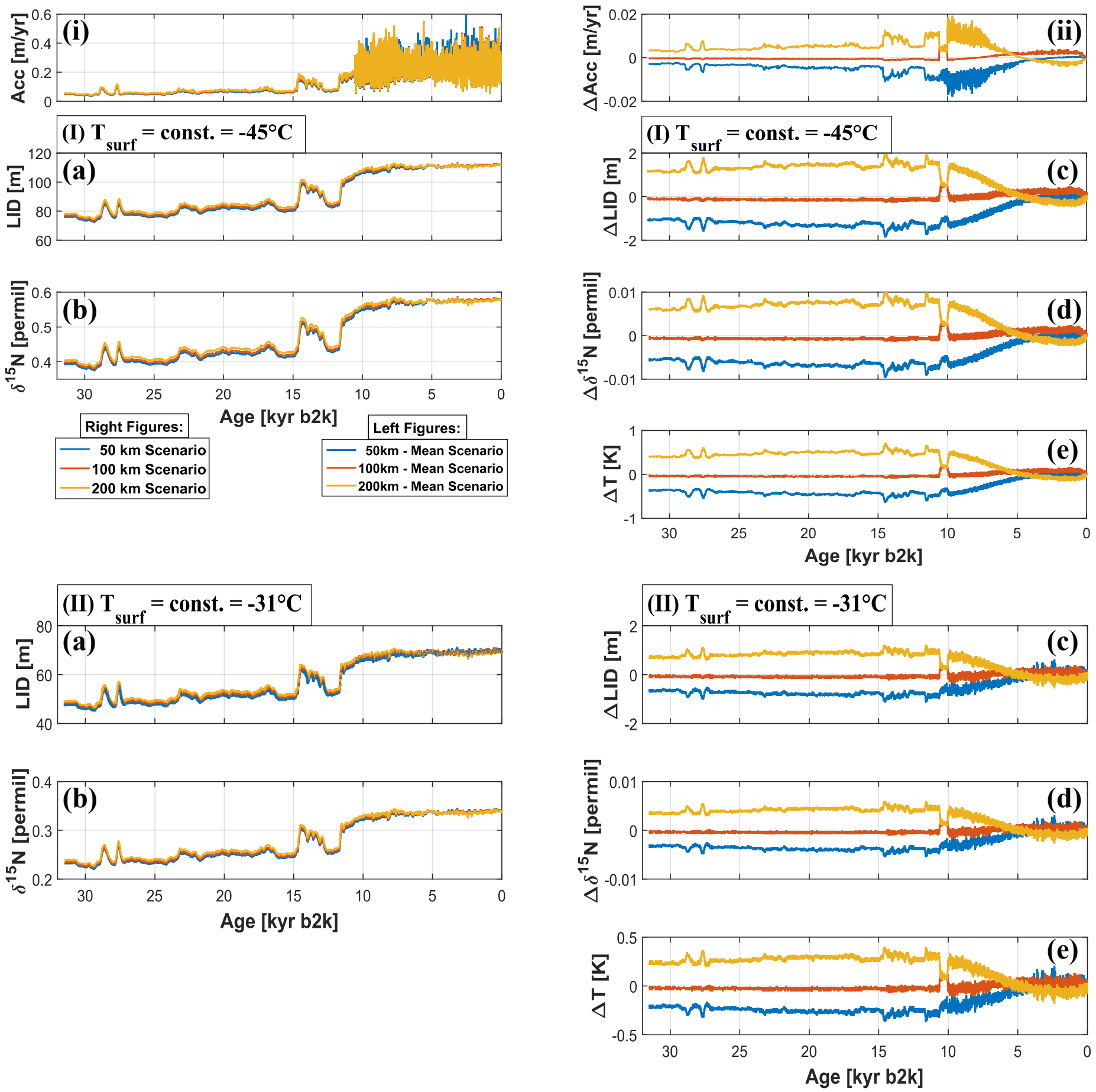

Figure S01: Possible accumulation rate scenarios (i), the deviation from the averaged scenario (ii) and resulting modelled lock-in-depth (LID) (a) and $\delta 15 \mathrm{~N}$ values (b) and deviations (c), (d), as well as, corresponding temperature uncertainties $\Delta \mathrm{T}$ (e), for a constant temperature input (I) $-45^{\circ} \mathrm{C}$ and (II) $-31^{\circ} \mathrm{C}$. 

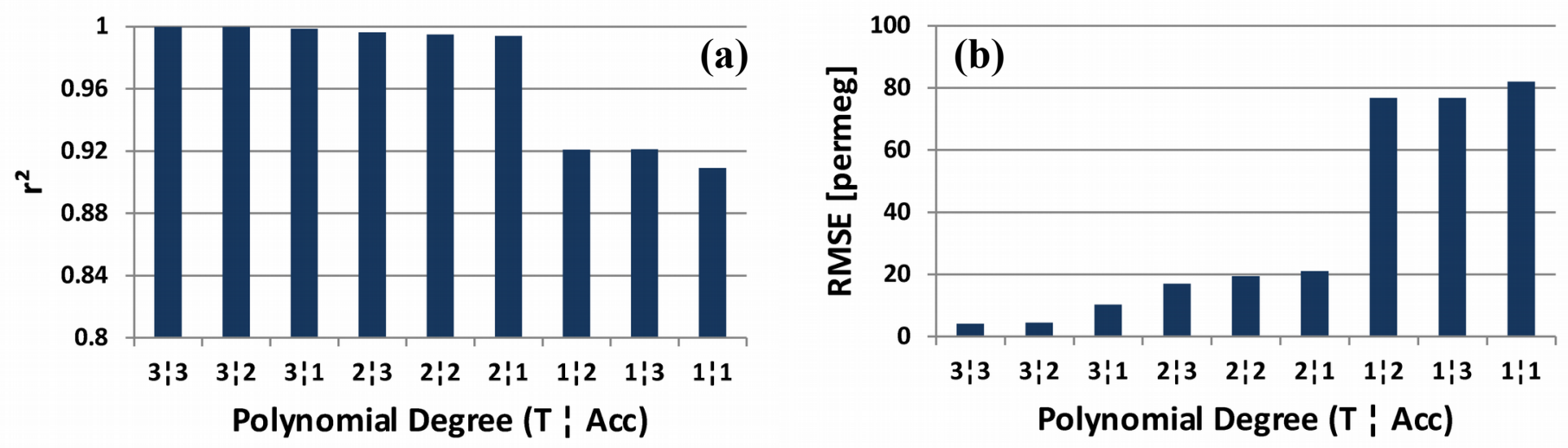

Figure S02: Static model behaviour: Two-dimensional (temperature T, accumulation rate Acc) polynomial surface fits. Correlation coefficients (a) and root mean squared error (b) versus polynomial degrees in temperature ( $T$ ) and accumulation rate (Acc). 

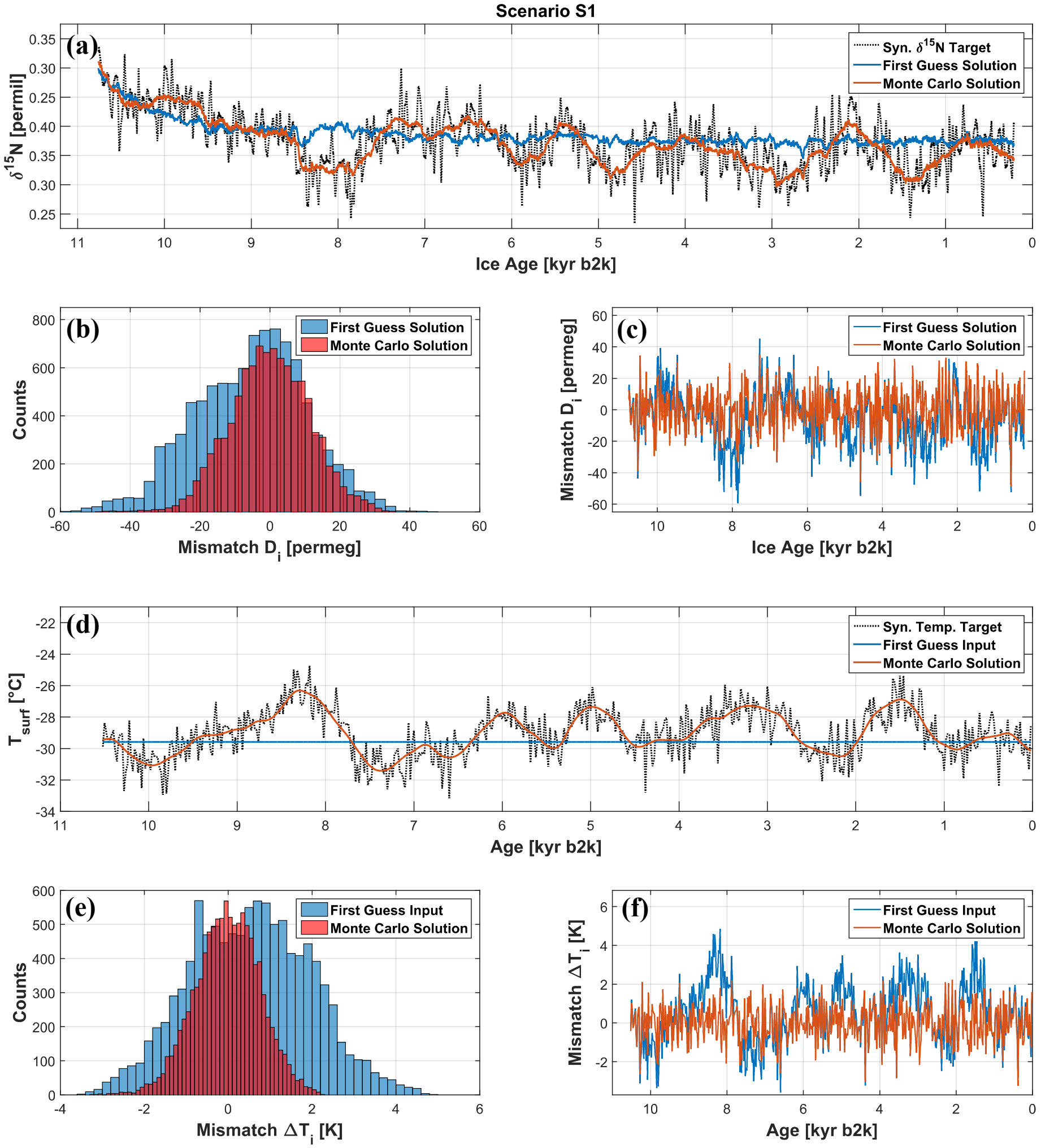

Figure S03: (a-c) First guess vs. Monte Carlo $\delta^{15} \mathrm{~N}$ solution for the scenario S1; (a) Synthetic $\delta^{15} \mathrm{~N}$ target (black dotted line), modelled $\delta^{15} \mathrm{~N}$ time series for the first guess input (blue line) and Monte Carlo solution (red line);

(b) Histogram shows the pointwise mismatches $D_{i}$ for the first guess solution (blue) and the Monte Carlo solution (red) versus the synthetic target; (c) Time series for the pointwise mismatches $D_{i}$ for the first guess solution (blue) and the Monte Carlo solution (red) versus the synthetic target; (d-f) First guess vs. Monte Carlo surface temperature solution for the scenario S1; (d) Synthetic surface temperature target (black dotted line), first guess temperature input (blue line) and Monte Carlo solution (red line); (e) Histogram shows the pointwise mismatches $\Delta \mathbf{T}_{\mathbf{i}}$ for the first guess solution (blue) and the Monte Carlo solution (red) versus the synthetic surface temperature target; (f) Time series for the pointwise mismatches $\Delta T_{i}$ for the first guess solution (blue) and the Monte Carlo solution (red) versus the synthetic surface temperature target; 

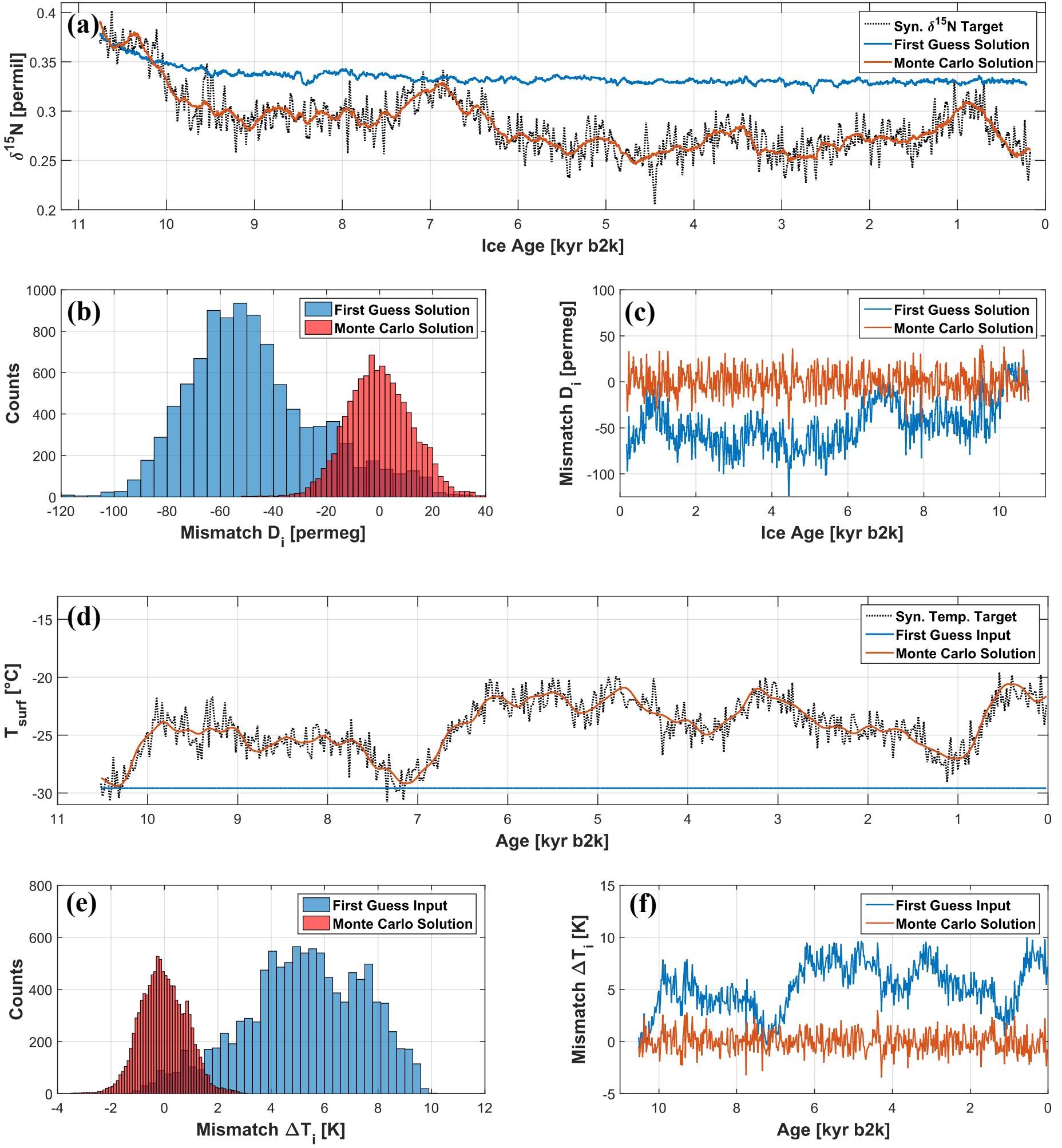

Figure S04: (a-c) First guess vs. Monte Carlo $\delta^{15} \mathrm{~N}$ solution for the scenario S2; (a) Synthetic $\delta^{15} \mathrm{~N}$ target (black dotted line), modelled $\delta^{15} \mathrm{~N}$ time series for the first guess input (blue line) and Monte Carlo solution (red line); (b) Histogram shows the pointwise mismatches $D_{i}$ for the first guess solution (blue) and the Monte Carlo solution (red) versus the synthetic target; (c) Time series for the pointwise mismatches $D_{i}$ for the first guess solution (blue) and the Monte Carlo solution (red) versus the synthetic target; (d-f) First guess vs. Monte Carlo surface temperature solution for the scenario S2; (d) Synthetic surface temperature target (black dotted line), first guess temperature input (blue line) and Monte Carlo solution (red line). (e) Histogram shows the pointwise mismatches $\Delta T_{i}$ for the first guess solution (blue) and the Monte Carlo solution (red) versus the synthetic surface temperature target; (f) Time series for the pointwise mismatches $\Delta T_{i}$ for the first guess solution (blue) and the Monte Carlo solution (red) versus the synthetic surface temperature target; 

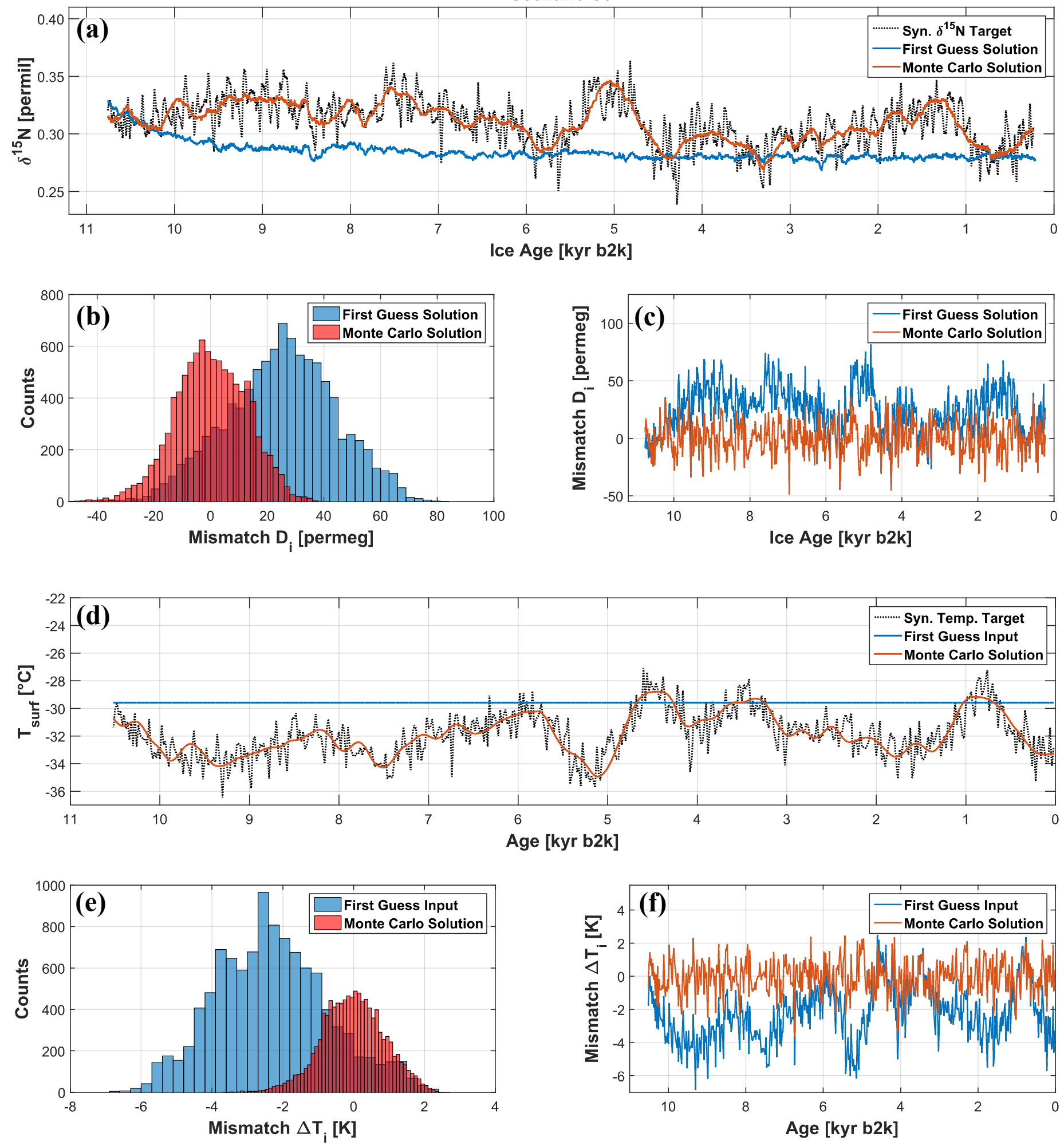

Figure S05: (a-c) First guess vs. Monte Carlo $\delta^{15} \mathrm{~N}$ solution for the scenario S3; (a) Synthetic $\delta^{15} \mathrm{~N}$ target (black dotted line), modelled $\delta^{15} \mathrm{~N}$ time series for the first guess input (blue line) and Monte Carlo solution (red line); (b) Histogram shows the pointwise mismatches $D_{i}$ for the first guess solution (blue) and the Monte Carlo solution (red) versus the synthetic target; (c) Time series for the pointwise mismatches $D_{i}$ for the first guess solution (blue) and the Monte Carlo solution (red) versus the synthetic target; (d-f) First guess vs. Monte Carlo surface temperature solution for the scenario S3; (d) Synthetic surface temperature target (black dotted line), first guess temperature input (blue line) and Monte Carlo solution (red line). (e) Histogram shows the pointwise mismatches $\Delta \mathbf{T}_{\mathbf{i}}$ for the first guess solution (blue) and the Monte Carlo solution (red) versus the synthetic surface temperature target; (f) Time series for the pointwise mismatches $\Delta T_{i}$ for the first guess solution (blue) and the Monte Carlo solution (red) versus the synthetic surface temperature target; 


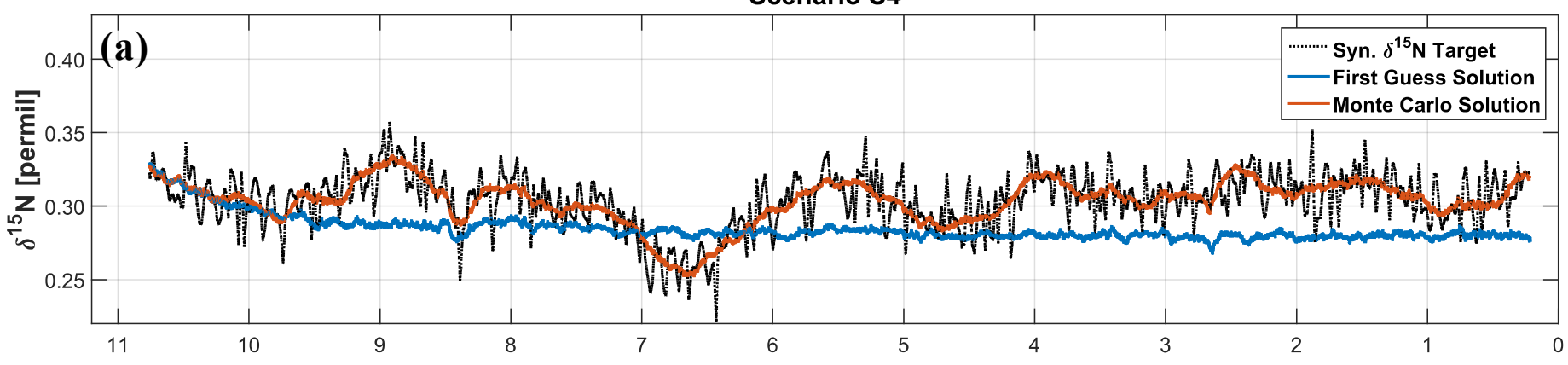

Ice Age [kyr b2k]
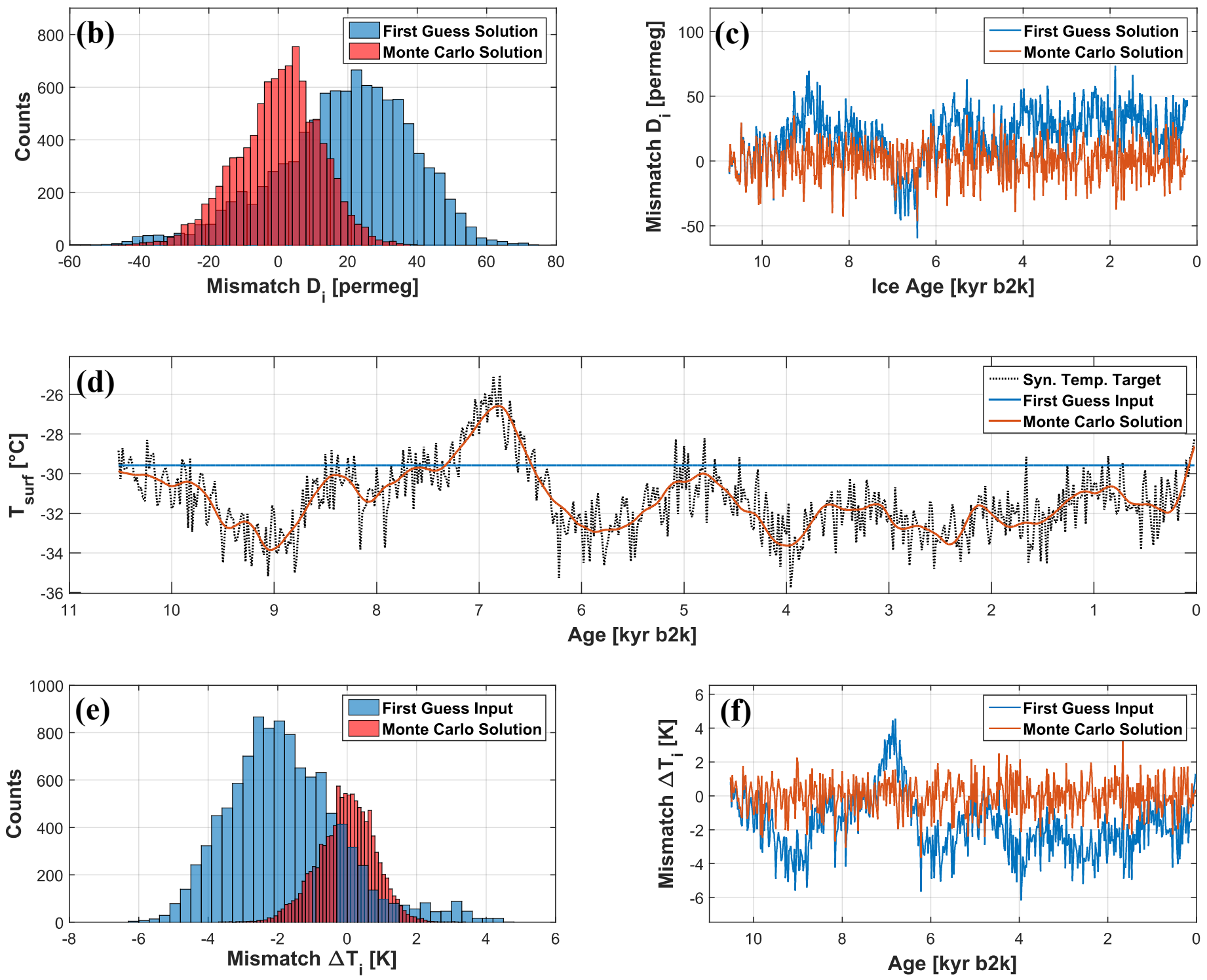

Figure S06: (a-c) First guess vs. Monte Carlo $\delta^{15} \mathrm{~N}$ solution for the scenario S4; (a) Synthetic $\delta^{15} \mathrm{~N}$ target (black dotted line), modelled $\delta^{15} \mathrm{~N}$ time series for the first guess input (blue line) and Monte Carlo solution (red line);

(b) Histogram shows the pointwise mismatches $D_{i}$ for the first guess solution (blue) and the Monte Carlo solution (red) versus the synthetic target; (c) Time series for the pointwise mismatches $D_{i}$ for the first guess solution (blue) and the Monte Carlo solution (red) versus the synthetic target; (d-f) First guess vs. Monte Carlo surface temperature solution for the scenario S4; (d) Synthetic surface temperature target (black dotted line), first guess temperature input (blue line) and Monte Carlo solution (red line). (e) Histogram shows the pointwise mismatches $\Delta \mathbf{T}_{\mathbf{i}}$ for the first guess solution (blue) and the Monte Carlo solution (red) versus the synthetic surface temperature target; (f) Time series for the pointwise mismatches $\Delta \mathbf{T}_{i}$ for the first guess solution (blue) and the Monte Carlo solution (red) versus the synthetic surface temperature target; 

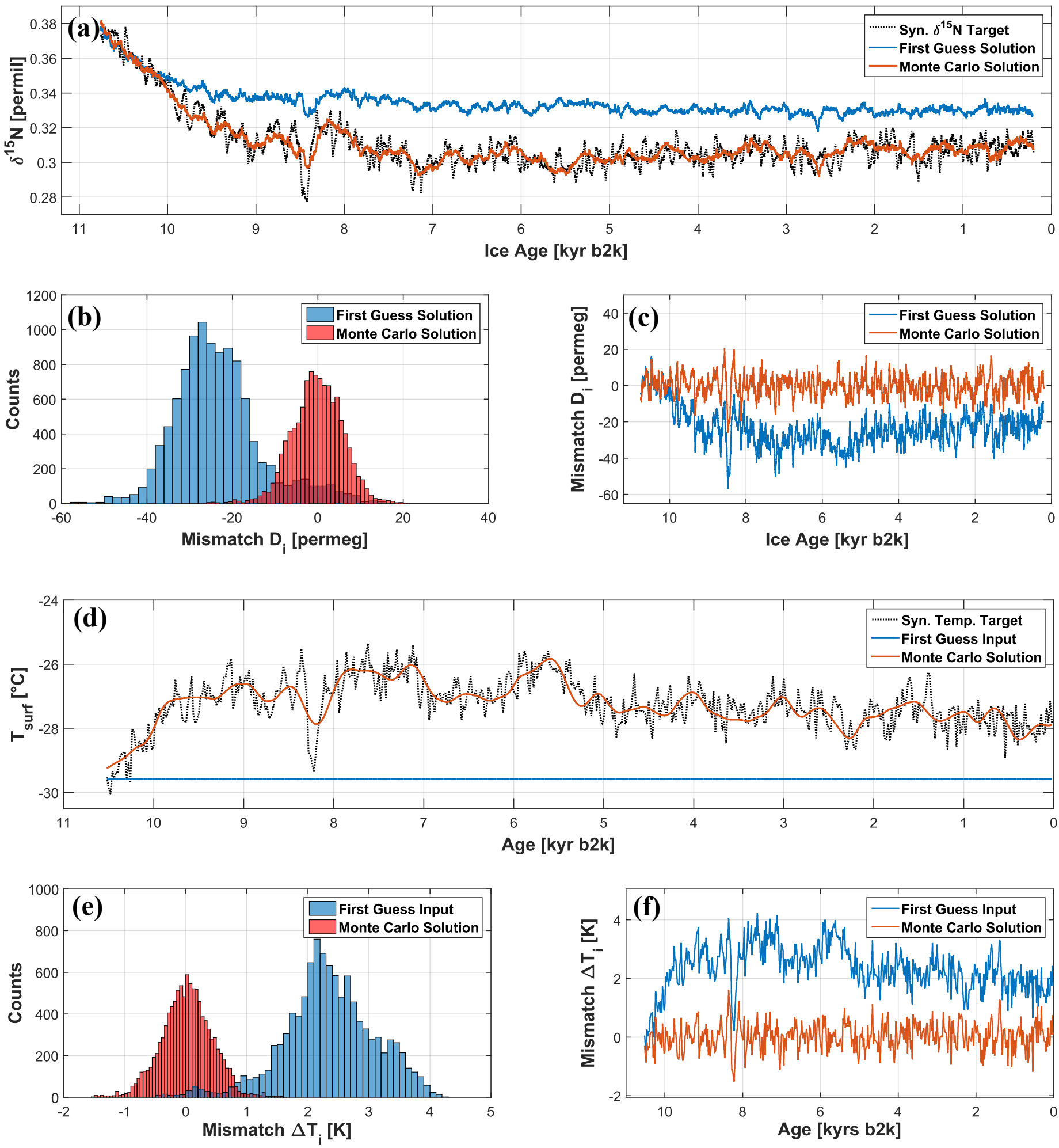

Figure S07: (a-c) First guess vs. Monte Carlo $\delta 15 \mathrm{~N}$ solution for the scenario H1; (a) Synthetic $\delta^{15} \mathrm{~N}$ target (black dotted line), modelled $\delta^{15} \mathrm{~N}$ time series for the first guess input (blue line) and Monte Carlo solution (red line); (b) Histogram shows the pointwise mismatches $D_{i}$ for the first guess solution (blue) and the Monte Carlo solution (red) versus the synthetic target; (c) Time series for the pointwise mismatches $D_{i}$ for the first guess solution (blue) and the Monte Carlo solution (red) versus the synthetic target; (d-f) First guess vs. Monte Carlo surface temperature solution for the scenario H1; (d) Synthetic surface temperature target (black dotted line), first guess temperature input (blue line) and Monte Carlo solution (red line). (e) Histogram shows the pointwise mismatches $\Delta \mathbf{T}_{\mathrm{i}}$ for the first guess solution (blue) and the Monte Carlo solution (red) versus the synthetic surface temperature target; (f) Time series for the pointwise mismatches $\Delta T_{i}$ for the first guess solution (blue) and the Monte Carlo solution (red) versus the synthetic surface temperature target; 

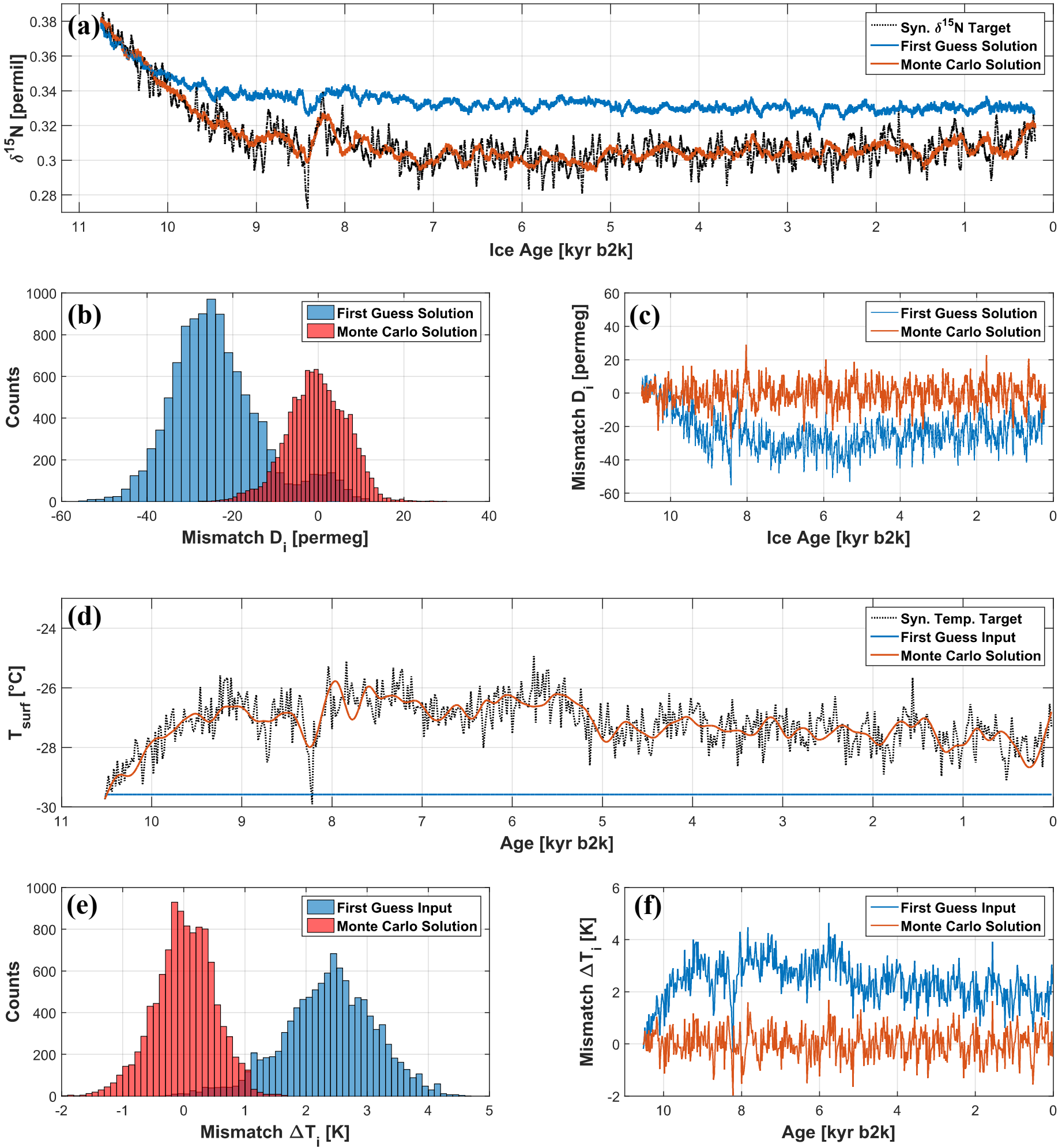

Figure S08: (a-c) First guess vs. Monte Carlo $\delta^{15} \mathrm{~N}$ solution for the scenario H2; (a) Synthetic $\delta^{15} \mathrm{~N}$ target (black dotted line), modelled $\delta^{15} \mathrm{~N}$ time series for the first guess input (blue line) and Monte Carlo solution (red line); (b) Histogram shows the pointwise mismatches $D_{i}$ for the first guess solution (blue) and the Monte Carlo solution (red) versus the synthetic target; (c) Time series for the pointwise mismatches $D_{i}$ for the first guess solution (blue) and the Monte Carlo solution (red) versus the synthetic target; (d-f) First guess vs. Monte Carlo surface temperature solution for the scenario H2; (d) Synthetic surface temperature target (black dotted line), first guess temperature input (blue line) and Monte Carlo solution (red line). (e) Histogram shows the pointwise mismatches $\Delta T_{i}$ for the first guess solution (blue) and the Monte Carlo solution (red) versus the synthetic surface temperature target; (f) Time series for the pointwise mismatches $\Delta T_{i}$ for the first guess solution (blue) and the Monte Carlo solution (red) versus the synthetic surface temperature target; 

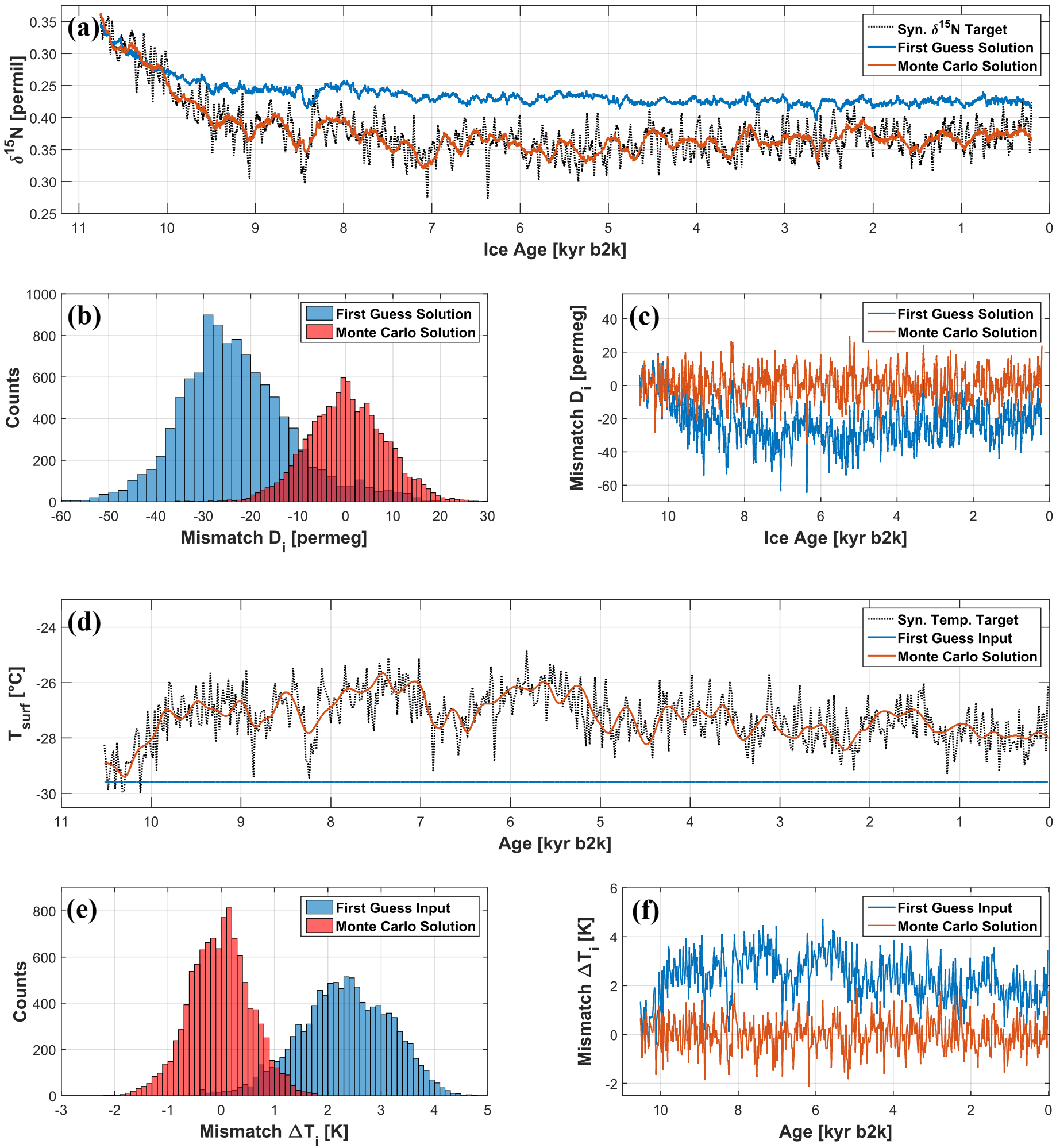

Figure S09: (a-c) First guess vs. Monte Carlo $\delta^{15} \mathrm{~N}$ solution for the scenario H3; (a) Synthetic $\delta^{15} \mathrm{~N}$ target (black dotted line), modelled $\delta^{15} \mathrm{~N}$ time series for the first guess input (blue line) and Monte Carlo solution (red line); (b) Histogram shows the pointwise mismatches $D_{i}$ for the first guess solution (blue) and the Monte Carlo solution (red) versus the synthetic target; (c) Time series for the pointwise mismatches $D_{i}$ for the first guess solution (blue) and the Monte Carlo solution (red) versus the synthetic target; (d-f) First guess vs. Monte Carlo surface temperature solution for the scenario S5; (d) Synthetic surface temperature target (black dotted line), first guess temperature input (blue line) and Monte Carlo solution (red line). (e) Histogram shows the pointwise mismatches $\Delta \mathbf{T}_{\mathrm{i}}$ for the first guess solution (blue) and the Monte Carlo solution (red) versus the synthetic surface temperature target; (f) Time series for the pointwise mismatches $\Delta T_{i}$ for the first guess solution (blue) and the Monte Carlo solution (red) versus the synthetic surface temperature target; 

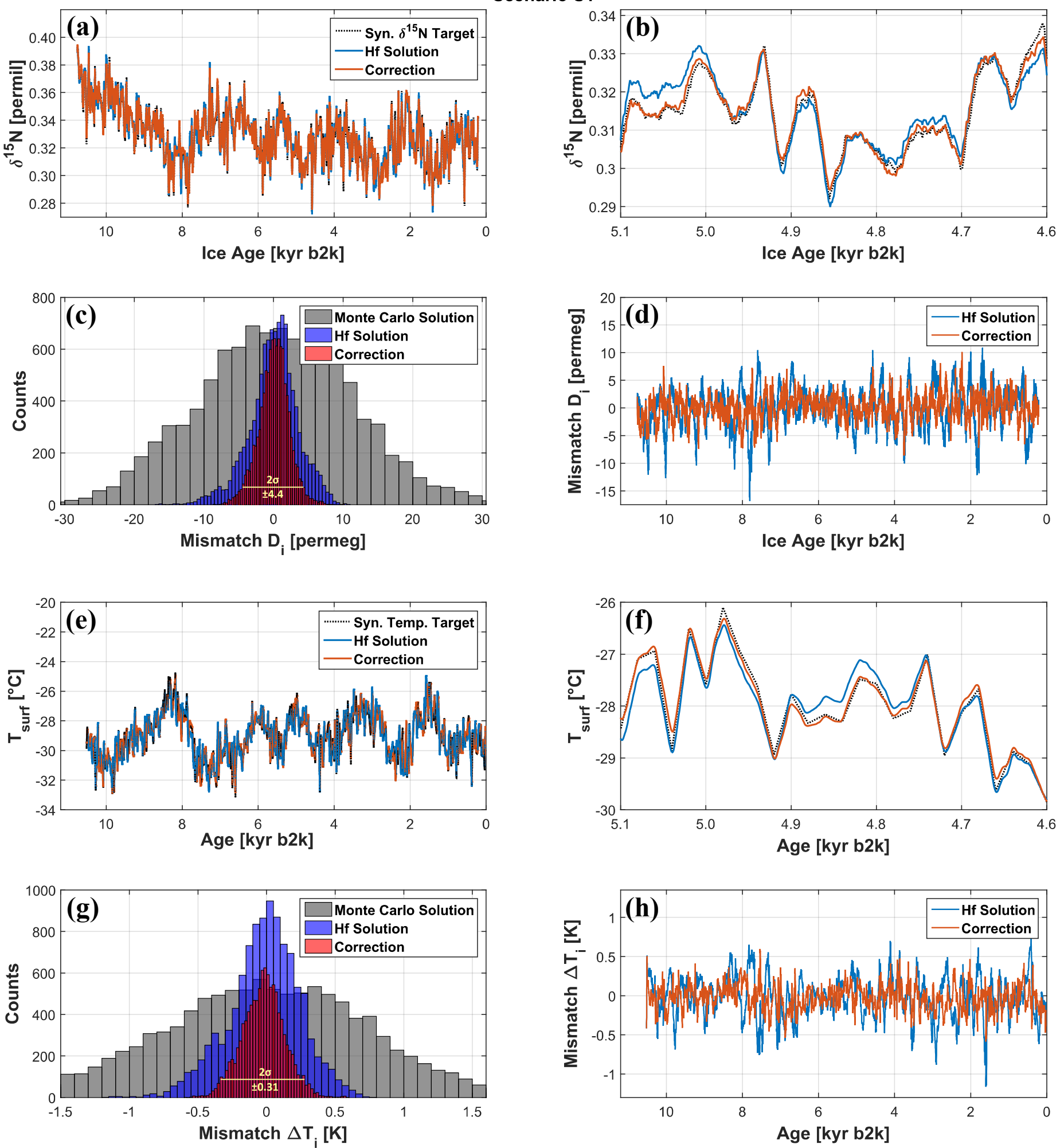

Figure S10: (a-d): (a) Synthetic $\delta^{15} \mathrm{~N}$ target (black dotted line), modelled $\delta^{15} \mathrm{~N}$ time series after adding high frequency information (blue line) and correction (red line) for the scenario S1; (b) Zoom in for a randomly chosen $500 \mathrm{yr}$ interval shows the decrease of the mismatch after the correction compared to the high frequency solution; (c) Histogram shows the pointwise mismatches $D_{i}$ from the synthetic $\delta^{15} \mathrm{~N}$ target for the Monte Carlo solution (grey), the high frequency solution (blue) and the correction (red); The $95 \%$ quantile is 4.4 permeg (yellow line) and used as an estimate for $2 \sigma$ uncertainty of the final solution; (d) Time series for the pointwise mismatches $D_{i}$ from the synthetic $\delta^{15} \mathrm{~N}$ target for the high frequency solution (blue) and the correction (red); (e-h): (e) Synthetic temperature target (black dotted line), modelled temperature time series after adding high frequency information (blue line) and correction (red line); (f) Zoom in for a randomly chosen $500 \mathrm{yr}$ interval shows the decrease of the mismatch after the correction compared to the high frequency solution; (g) Histogram shows the pointwise mismatches $\Delta T_{i}$ from the synthetic temperature target for the Monte Carlo solution (grey), the high frequency solution (blue) and the correction (red); The $95 \%$ quantile is $0.31 \mathrm{~K}$ (yellow line) and used as an estimate for $2 \sigma$ uncertainty of the final solution; (h) Time series for the pointwise mismatches $\Delta T_{i}$ from the synthetic temperature target for the high frequency solution (blue) and the correction (red); 

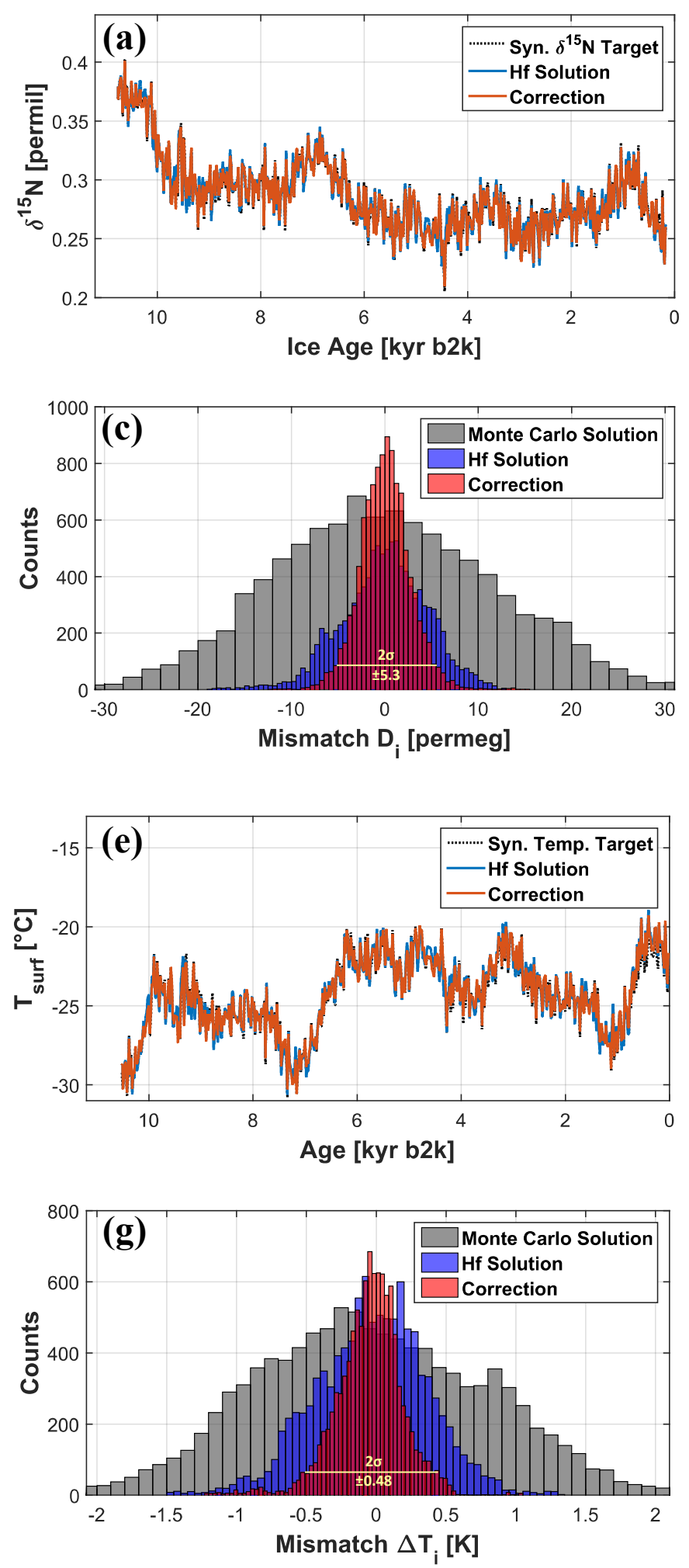
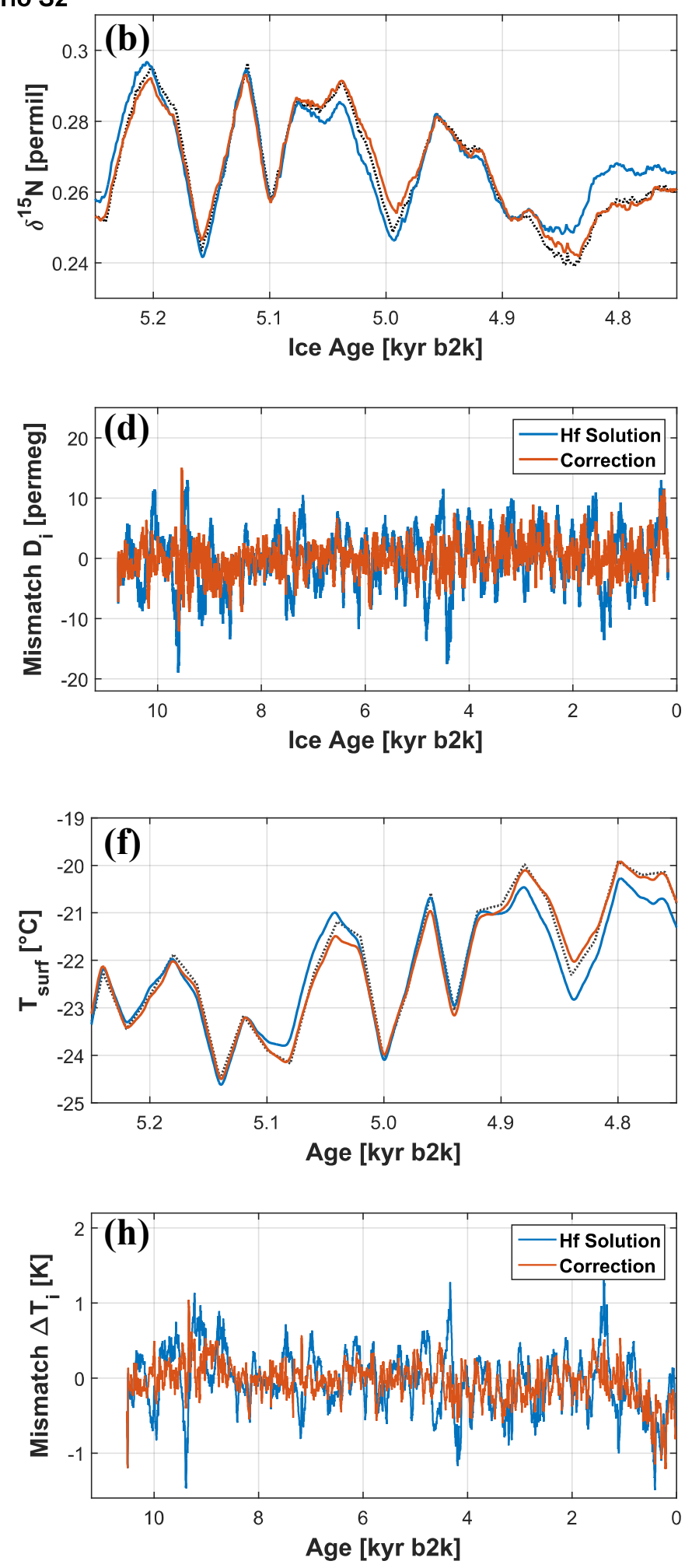

Figure S11: (a-d): (a) Synthetic $\delta^{15} \mathrm{~N}$ target (black dotted line), modelled $\delta^{15} \mathrm{~N}$ time series after adding high frequency information (blue line) and correction (red line) for the scenario S2; (b) Zoom in for a randomly chosen $500 \mathrm{yr}$ interval shows the decrease of the mismatch after the correction compared to the high frequency solution; (c) Histogram shows the pointwise mismatches $D_{i}$ from the synthetic $\delta^{15} \mathbf{N}$ target for the Monte Carlo solution (grey), the high frequency solution (blue) and the correction (red); The $95 \%$ quantile is 5.3 permeg (yellow line) and used as an estimate for $2 \sigma$ uncertainty of the final solution; (d) Time series for the pointwise mismatches $D_{i}$ from the synthetic $\delta^{15} \mathrm{~N}$ target for the high frequency solution (blue) and the correction (red); (e-h): (e) Synthetic temperature target (black dotted line), modelled temperature time series after adding high frequency information (blue line) and correction (red line); (f) Zoom in for a randomly chosen $500 \mathrm{yr}$ interval shows the decrease of the mismatch after the correction compared to the high frequency solution; (g) Histogram shows the pointwise mismatches $\Delta \mathbf{T}_{i}$ from the synthetic temperature target for the Monte Carlo solution (grey), the high frequency solution (blue) and the correction (red); The $95 \%$ quantile is $0.48 \mathrm{~K}$ (yellow line) and used as an estimate for $2 \sigma$ uncertainty of the final solution; (h) Time series for the pointwise mismatches $\Delta T_{i}$ from the synthetic temperature target for the high frequency solution (blue) and the correction (red); 

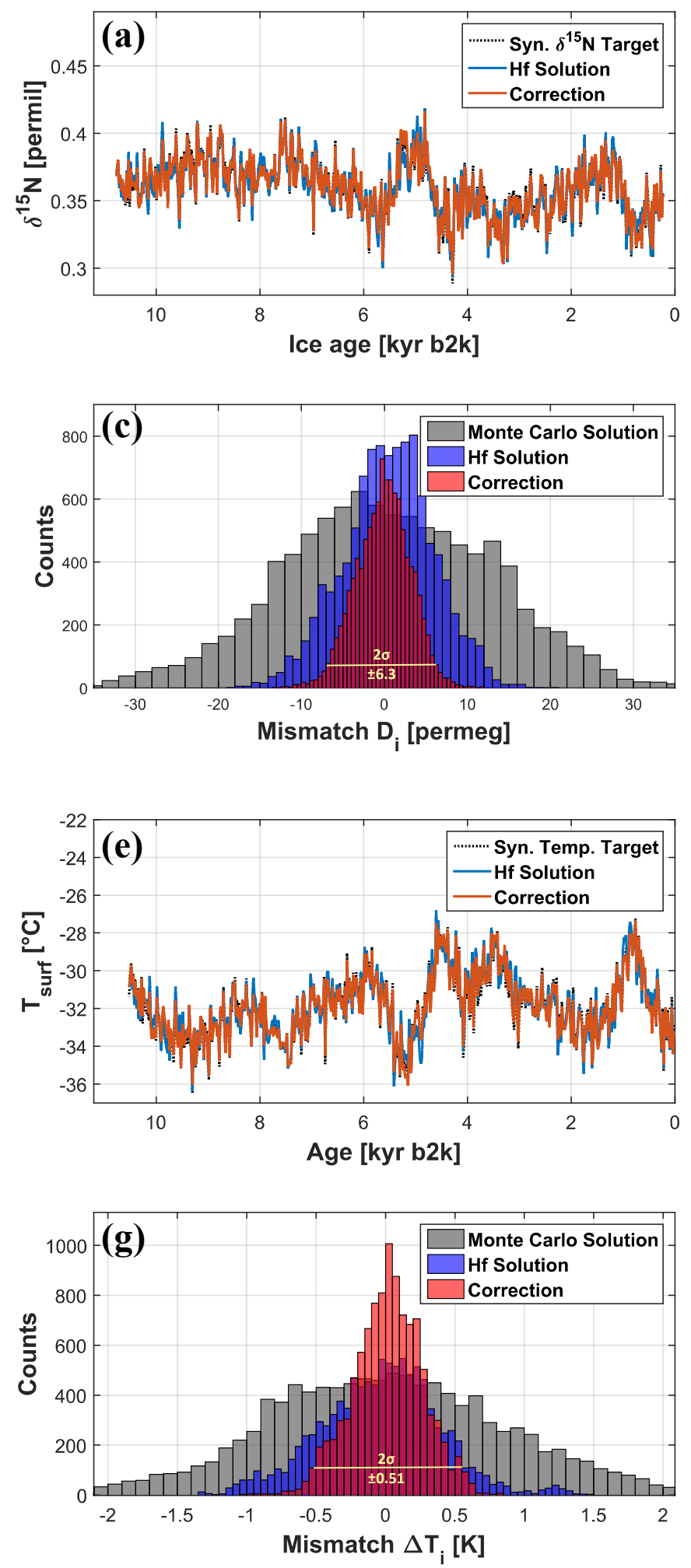
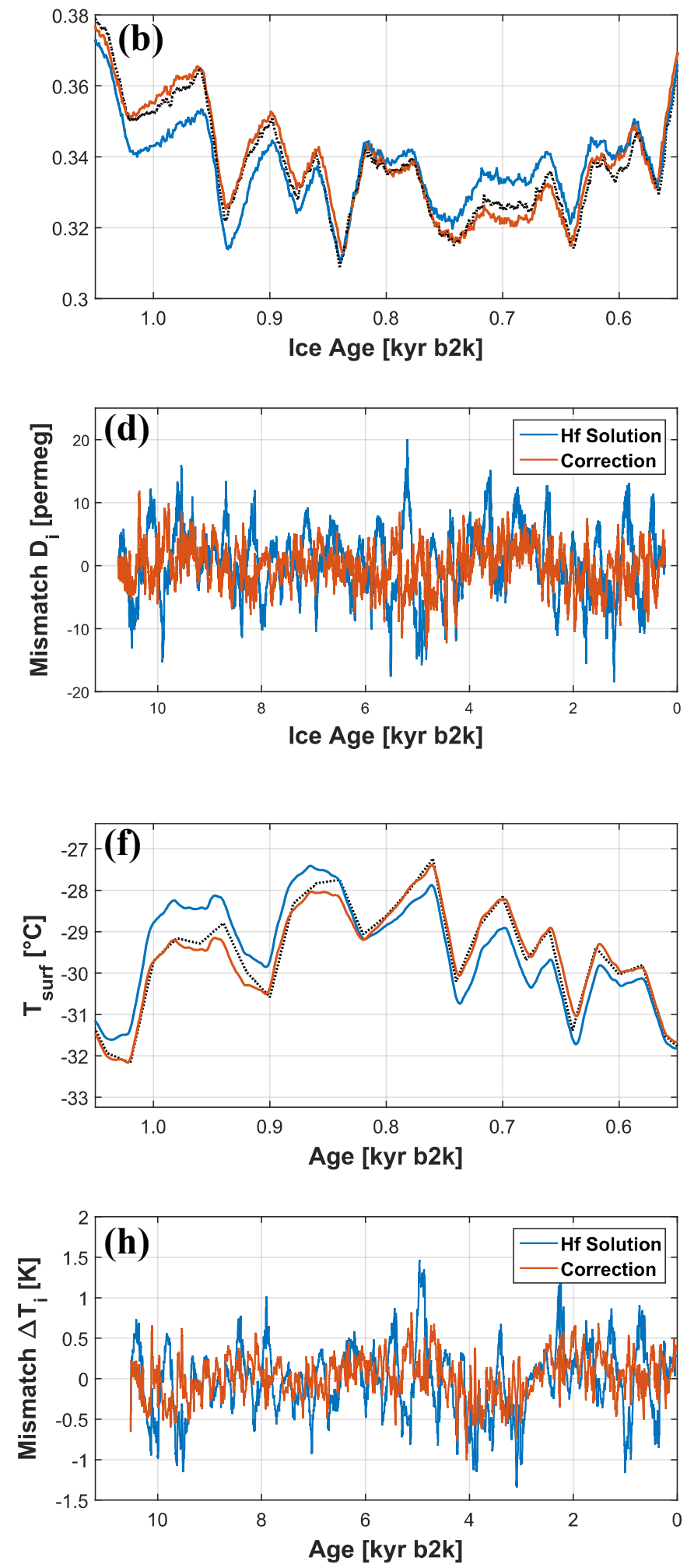

Figure S12: (a-d): (a) Synthetic $\delta^{15} \mathrm{~N}$ target (black dotted line), modelled $\delta^{15} \mathrm{~N}$ time series after adding high frequency information (blue line) and correction (red line) for the scenario S3; (b) Zoom in for a randomly chosen $500 \mathrm{yr}$ interval shows the decrease of the mismatch after the correction compared to the high frequency solution; (c) Histogram shows the pointwise mismatches $D_{i}$ from the synthetic $\delta 15 \mathrm{~N}$ target for the Monte Carlo solution (grey), the high frequency solution (blue) and the correction (red); The $95 \%$ quantile is 6.3 permeg (yellow line) and used as an estimate for $2 \sigma$ uncertainty of the final solution; (d) Time series for the pointwise mismatches $D_{i}$ from the synthetic $\delta^{15} \mathrm{~N}$ target for the high frequency solution (blue) and the correction (red); (e-h): (e) Synthetic temperature target (black dotted line), modelled temperature time series after adding high frequency information (blue line) and correction (red line); (f) Zoom in for a randomly chosen $500 \mathrm{yr}$ interval shows the decrease of the mismatch after the correction compared to the high frequency solution; (g) Histogram shows the pointwise mismatches $\Delta T_{i}$ from the synthetic temperature target for the Monte Carlo solution (grey), the high frequency solution (blue) and the correction (red); The $95 \%$ quantile is $0.51 \mathrm{~K}$ (yellow line) and used as an estimate for $2 \sigma$ uncertainty of the final solution; (h) Time series for the pointwise mismatches $\Delta T_{i}$ from the synthetic temperature target for the high frequency solution (blue) and the correction (red); 

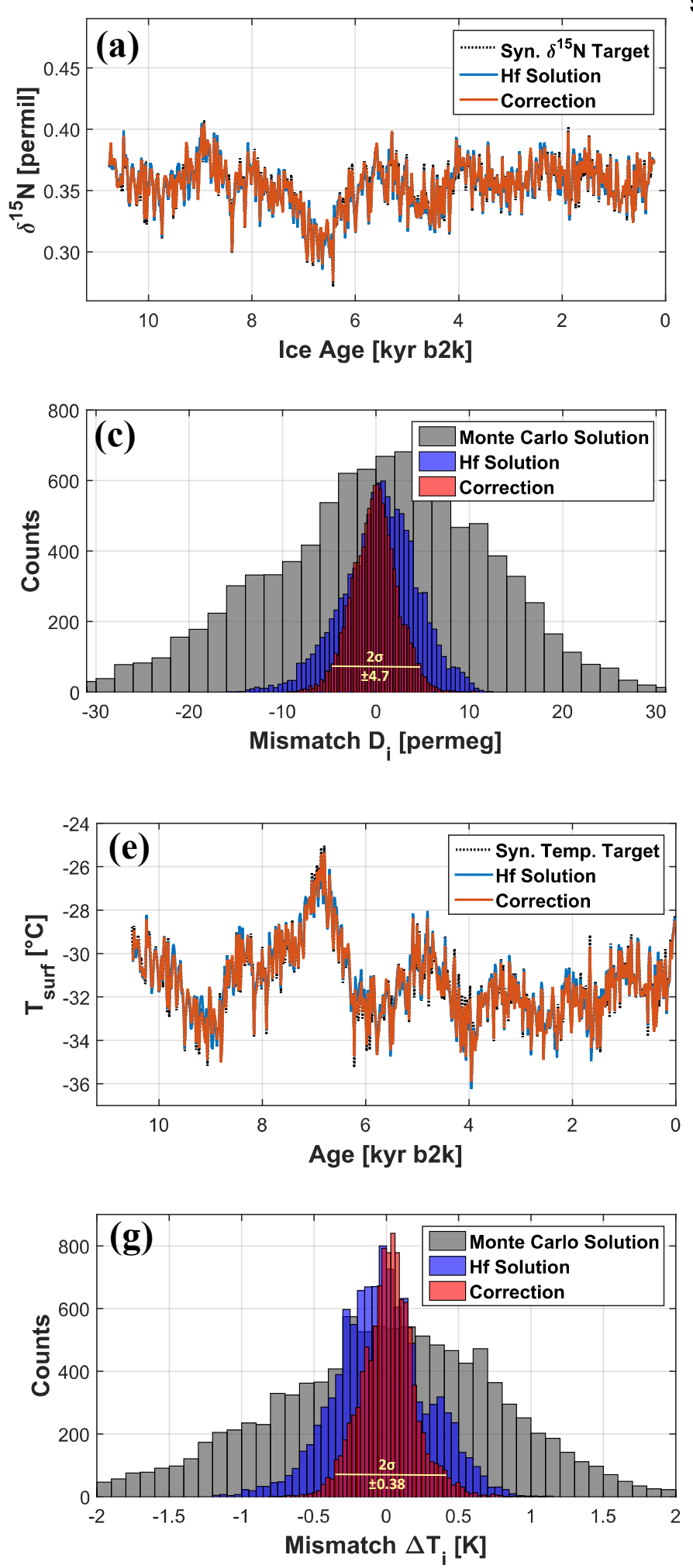

\section{Scenario 54}
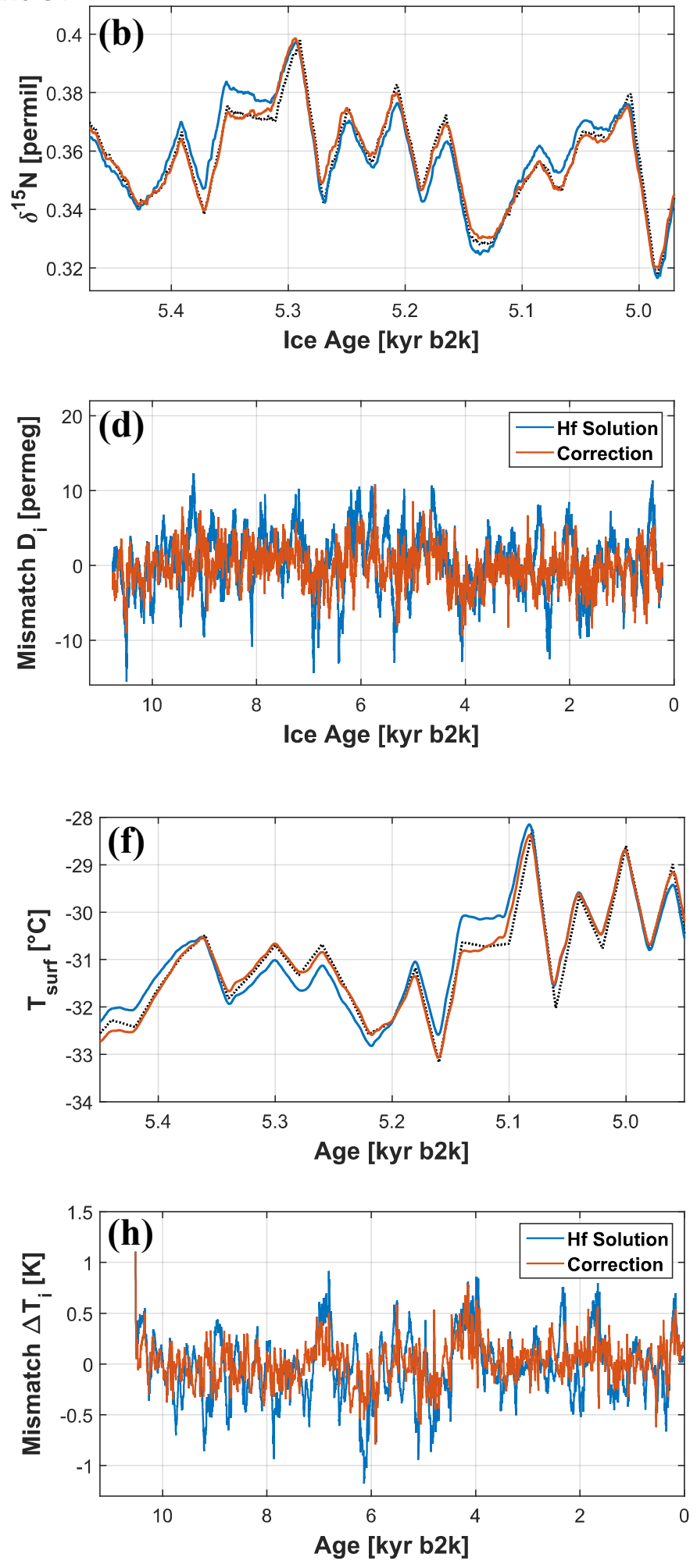

Figure S13: (a-d): (a) Synthetic $\delta^{15} \mathbf{N}$ target (black dotted line), modelled $\delta^{15} \mathbf{N}$ time series after adding high frequency information (blue line) and correction (red line) for the scenario S4; (b) Zoom in for a randomly chosen $500 \mathrm{yr}$ interval shows the decrease of the mismatch after the correction compared to the high frequency solution; (c) Histogram shows the pointwise mismatches $D_{i}$ from the synthetic $\delta^{15} \mathbf{N}$ target for the Monte Carlo solution (grey), the high frequency solution (blue) and the correction (red); The $95 \%$ quantile is 4.7 permeg (yellow line) and used as an estimate for $2 \sigma$ uncertainty of the final solution; (d) Time series for the pointwise mismatches $D_{i}$ from the synthetic $\delta^{15} \mathrm{~N}$ target for the high frequency solution (blue) and the correction (red); (e-h): (e) Synthetic temperature target (black dotted line), modelled temperature time series after adding high frequency information (blue line) and correction (red line); (f) Zoom in for a randomly chosen $500 \mathrm{yr}$ interval shows the decrease of the mismatch after the correction compared to the high frequency solution; (g) Histogram shows the pointwise mismatches $\Delta T_{i}$ from the synthetic temperature target for the Monte Carlo solution (grey), the high frequency solution (blue) and the correction (red); The $95 \%$ quantile is $0.38 \mathrm{~K}$ (yellow line) and used as an estimate for $2 \sigma$ uncertainty of the final solution; (h) Time series for the pointwise mismatches $\Delta T_{i}$ from the synthetic temperature target for the high frequency solution (blue) and the correction (red); 

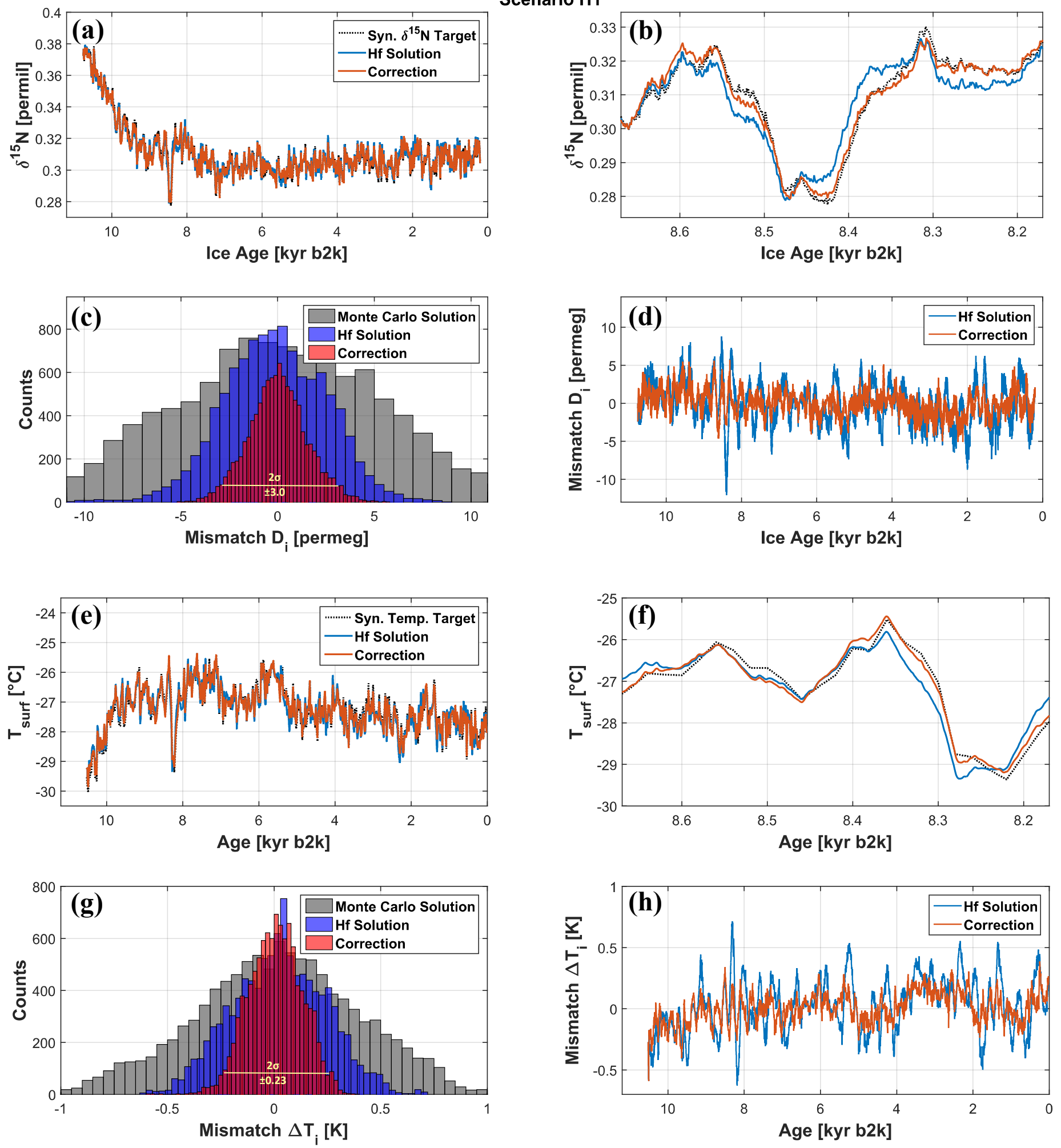

Figure S14: (a-d): (a) Synthetic $\delta^{15} \mathbf{N}$ target (black dotted line), modelled $\delta^{15} \mathrm{~N}$ time series after adding high frequency information (blue line) and correction (red line) for the scenario H1; (b) Zoom in for a randomly chosen $500 \mathrm{yr}$ interval shows the decrease of the mismatch after the correction compared to the high frequency solution; (c) Histogram shows the pointwise mismatches $D_{i}$ from the synthetic $\delta^{15} \mathrm{~N}$ target for the Monte Carlo solution (grey), the high frequency solution (blue) and the correction (red); The $95 \%$ quantile is 3.0 permeg (yellow line) and used as an estimate for $2 \sigma$ uncertainty of the final solution; (d) Time series for the pointwise mismatches $D_{i}$ from the synthetic $\delta^{15} \mathrm{~N}$ target for the high frequency solution (blue) and the correction (red); (e-h): (e) Synthetic temperature target (black dotted line), modelled temperature time series after adding high frequency information (blue line) and correction (red line); (f) Zoom in for a randomly chosen $500 \mathrm{yr}$ interval shows the decrease of the mismatch after the correction compared to the high frequency solution; (g) Histogram shows the pointwise mismatches $\Delta \mathbf{T}_{\mathbf{i}}$ from the synthetic temperature target for the Monte Carlo solution (grey), the high frequency solution (blue) and the correction (red); The $95 \%$ quantile is $0.23 \mathrm{~K}$ (yellow line) and used as an estimate for $2 \sigma$ uncertainty of the final solution; (h) Time series for the pointwise mismatches $\Delta T_{i}$ from the synthetic temperature target for the high frequency solution (blue) and the correction (red); 

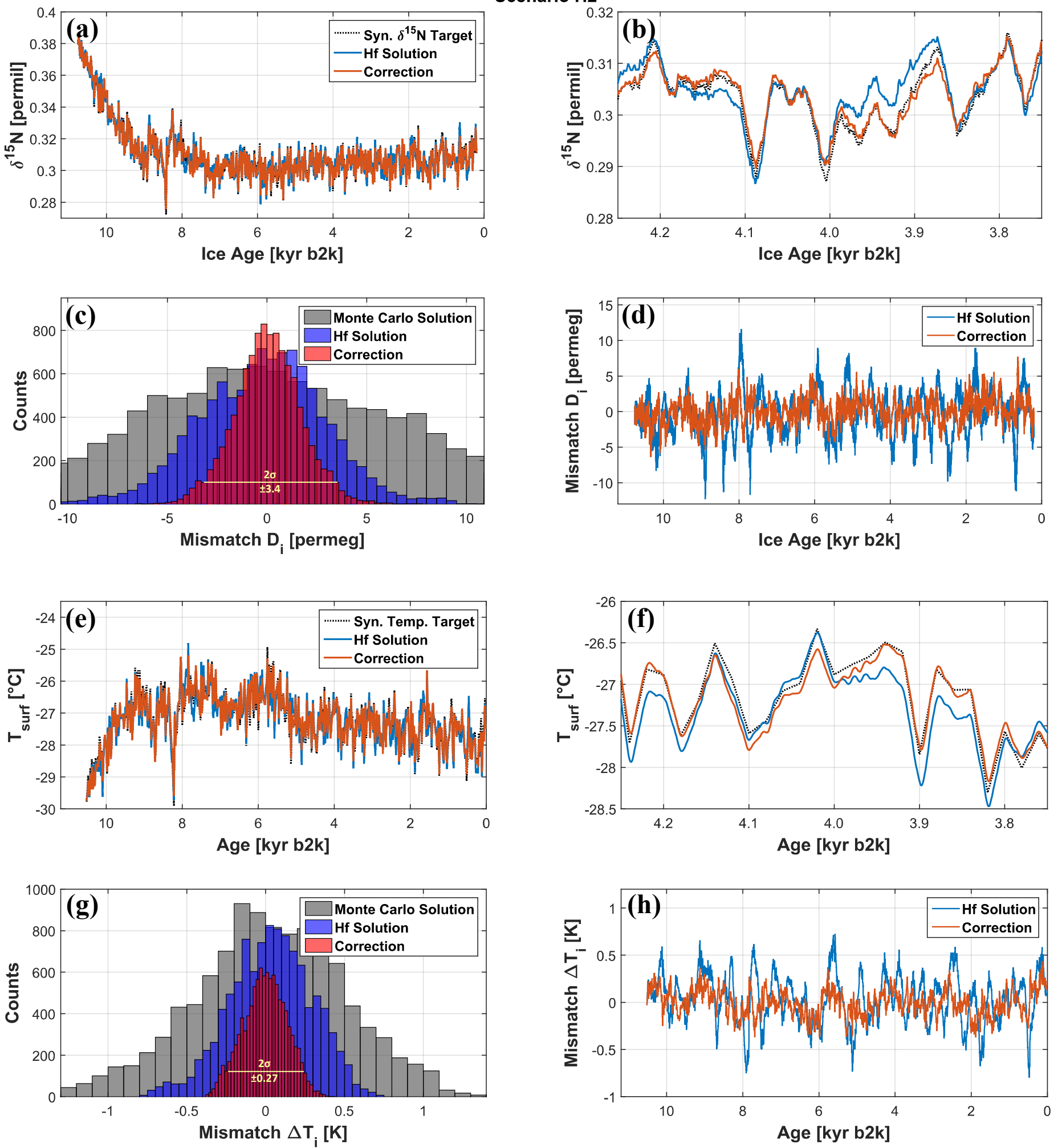

Figure S15: (a-d): (a) Synthetic $\delta^{15} \mathrm{~N}$ target (black dotted line), modelled $\delta^{15} \mathrm{~N}$ time series after adding high frequency information (blue line) and correction (red line) for the scenario $\mathrm{H2}$; (b) Zoom in for a randomly chosen 500 yr interval shows the decrease of the mismatch after the correction compared to the high frequency solution; (c) Histogram shows the pointwise mismatches $D_{i}$ from the synthetic $\delta^{15} \mathrm{~N}$ target for the Monte Carlo solution (grey), the high frequency solution (blue) and the correction (red); The $95 \%$ quantile is 3.4 permeg (yellow line) and used as an estimate for $2 \sigma$ uncertainty of the final solution; (d) Time series for the pointwise mismatches $D_{i}$ from the synthetic $\delta^{15} \mathbf{N}$ target for the high frequency solution (blue) and the correction (red); (e-h): (e) Synthetic temperature target (black dotted line), modelled temperature time series after adding high frequency information (blue line) and correction (red line); (f) Zoom in for a randomly chosen 500 yr interval shows the decrease of the mismatch after the correction compared to the high frequency solution; (g) Histogram shows the pointwise mismatches $\Delta \mathbf{T}_{\mathbf{i}}$ from the synthetic temperature target for the Monte Carlo solution (grey), the high frequency solution (blue) and the correction (red); The $95 \%$ quantile is $0.27 \mathrm{~K}$ (yellow line) and used as an estimate for $2 \sigma$ uncertainty of the final solution; (h) Time series for the pointwise mismatches $\Delta T_{i}$ from the synthetic temperature target for the high frequency solution (blue) and the correction (red); 
Scenario $\mathrm{H} 3$
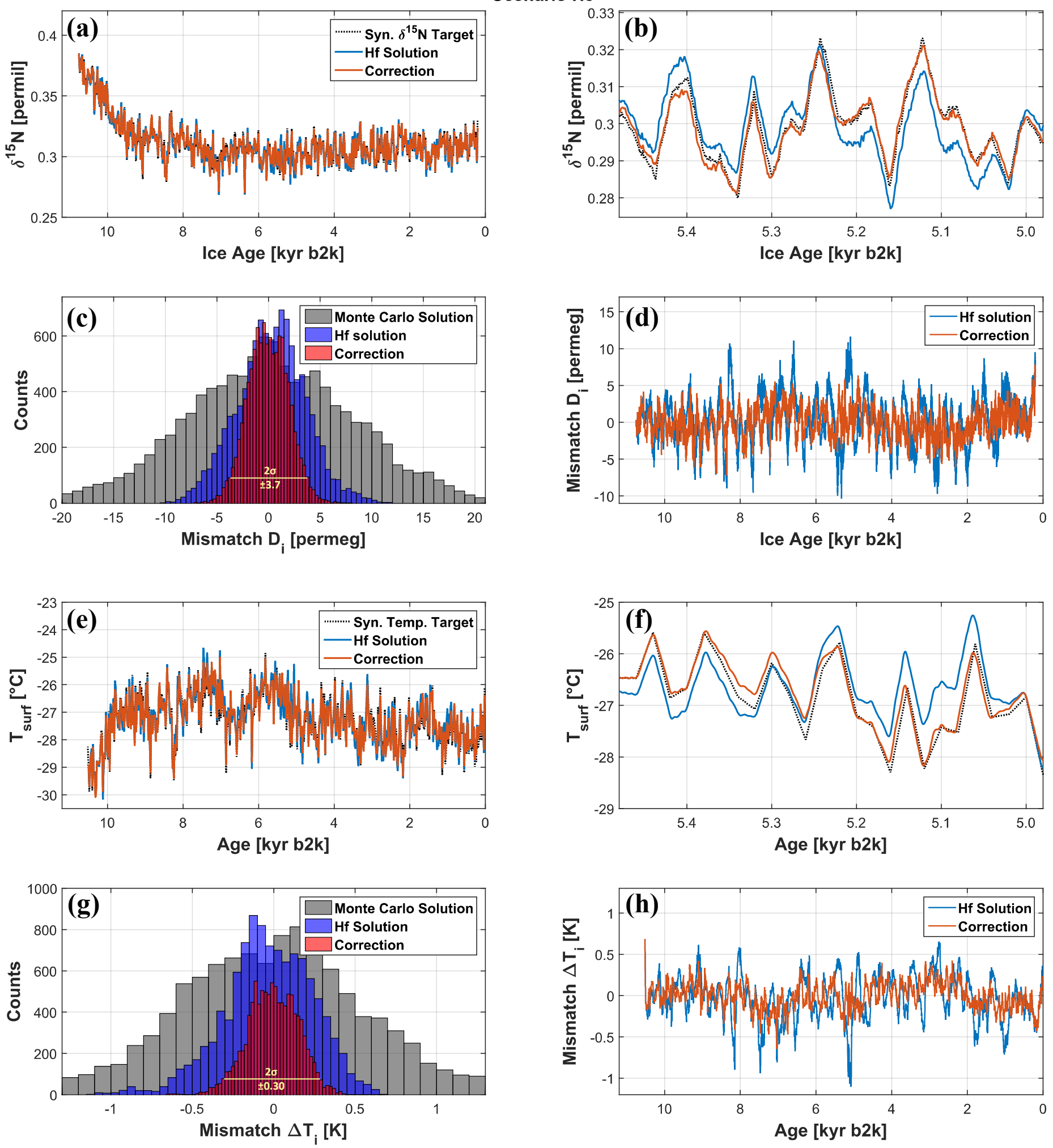

Figure S16: (a-d): (a) Synthetic $\delta^{15} \mathbf{N}$ target (black dotted line), modelled $\delta^{15} \mathbf{N}$ time series after adding high frequency information (blue line) and correction (red line) for the scenario $\mathrm{H3}$; (b) Zoom in for a randomly chosen $500 \mathrm{yr}$ interval shows the decrease of the mismatch after the correction compared to the high frequency solution; (c) Histogram shows the pointwise mismatches $D_{i}$ from the synthetic $\delta^{15} \mathrm{~N}$ target for the Monte Carlo solution (grey), the high frequency solution (blue) and the correction (red); The $95 \%$ quantile is 3.7 permeg (yellow line) and used as an estimate for $2 \sigma$ uncertainty of the final solution; (d) Time series for the pointwise mismatches $D_{i}$ from the synthetic $\delta^{15} \mathrm{~N}$ target for the high frequency solution (blue) and the correction (red); (e-h): (e) Synthetic temperature target (black dotted line), modelled temperature time series after adding high frequency information (blue line) and correction (red line); (f) Zoom in for a randomly chosen $500 \mathrm{yr}$ interval shows the decrease of the mismatch after the correction compared to the high frequency solution; (g) Histogram shows the pointwise mismatches $\Delta \mathbf{T}_{\mathrm{i}}$ from the synthetic temperature target for the Monte Carlo solution (grey), the high frequency solution (blue) and the correction (red); The $95 \%$ quantile is $0.30 \mathrm{~K}$ (yellow line) and used as an estimate for $2 \sigma$ uncertainty of the final solution; (h) Time series for the pointwise mismatches $\Delta \mathbf{T}_{\mathrm{i}}$ from the synthetic temperature target for the high frequency solution (blue) and the correction (red); 
Tables (supplementary):

\begin{tabular}{|c|c|c|c|c|c|}
\hline $\begin{array}{l}\text { Polynomial: } \\
\text { (T, Acc) }\end{array}$ & \multicolumn{2}{|c|}{$\begin{array}{l}\text { Coefficients } \\
\text { ( } 95 \% \text { confidence bounds) }\end{array}$} & Unit & $\mathbf{r}^{2}$ & $\begin{array}{l}\text { RMSE } \\
\text { [permeg] }\end{array}$ \\
\hline \multicolumn{6}{|l|}{ Static behaviour: } \\
\hline \multirow[b]{4}{*}{ 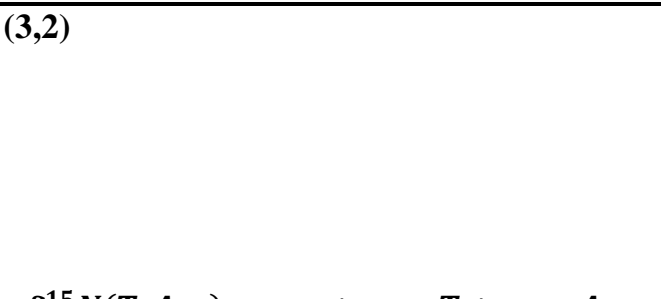 } & $p_{00}:$ & $\begin{array}{l}106.4 \\
(104,108.7)\end{array}$ & {$[\% 0]$} & \multirow[t]{9}{*}{0.9997} & \multirow[t]{9}{*}{4.5} \\
\hline & $p_{10}:$ & $\begin{array}{l}-1.253 \\
(-1.283,-1.224) \\
\end{array}$ & {$[\% / K]$} & & \\
\hline & $p_{01}:$ & $\begin{array}{l}29.16 \\
(27.99,30.32) \\
\end{array}$ & {$[y r \cdot \% 0 / m]$} & & \\
\hline & $p_{20}:$ & $\begin{array}{l}4.949 \cdot 10^{-3} \\
(4.824,5.074) \cdot 10^{-3}\end{array}$ & {$\left[\% / K^{2}\right]$} & & \\
\hline \multirow{5}{*}{$\begin{aligned} \delta^{15} N(T, A c c)= & p_{00}+p_{10} \cdot T+p_{01} \cdot A c c \\
& +p_{20} \cdot T^{2}+p_{02} \cdot A c c^{2} \\
& +p_{30} \cdot T^{3}+p_{11} \cdot T \cdot A c c \\
& +p_{21} \cdot T^{2} \cdot A c c+p_{12} \cdot T \\
& \cdot A c c^{2}\end{aligned}$} & $p_{02}:$ & $\begin{array}{l}-6.887 \\
(-7.505,-6.268) \\
\end{array}$ & {$\left[\frac{y r^{2} \cdot \% 0}{m^{2}}\right]$} & & \\
\hline & $p_{30}:$ & $\begin{array}{l}-6.536 \cdot 10^{-6} \\
(-6.711,-6.361) \cdot 10^{-6}\end{array}$ & {$\left[\% / K^{3}\right]$} & & \\
\hline & $p_{11}:$ & $\begin{array}{l}-0.2075 \\
(-0.217,-0.198) \\
\end{array}$ & {$\left[\frac{y r \cdot \% 0}{m \cdot K}\right]$} & & \\
\hline & $p_{21}:$ & $\begin{array}{l}3.707 \cdot 10^{-4} \\
(3.51,3.904) \cdot 10^{-4}\end{array}$ & {$\left[\frac{y r \cdot \% 0}{m \cdot K^{2}}\right]$} & & \\
\hline & $p_{12}:$ & $\begin{array}{l}0.02594 \\
(0.02335,0.02853)\end{array}$ & $\frac{y r^{2} \cdot \% 0}{m^{2} \cdot K}$ & & \\
\hline \multicolumn{6}{|l|}{ Dynamic behaviour: } \\
\hline \multirow[t]{4}{*}{$(3,2)$} & $p_{00}:$ & $\begin{array}{l}-1697 \\
(-1821,-1574) \\
\end{array}$ & {$[\%$} & \multirow[t]{9}{*}{0.6995} & \multirow[t]{9}{*}{34.2} \\
\hline & $p_{10}:$ & $\begin{array}{l}23.07 \\
(21.44,24.71) \\
\end{array}$ & {$[\% / K]$} & & \\
\hline & $p_{01}:$ & $\begin{array}{l}-2221 \\
(-2332,-2110) \\
\end{array}$ & {$[y r \cdot \% 0 / m]$} & & \\
\hline & $p_{20}:$ & $\begin{array}{l}-0.1044 \\
(-0.1116,-0.09714)\end{array}$ & {$\left[\% 0 / K^{2}\right]$} & & \\
\hline \multirow{5}{*}{$\begin{aligned} \delta^{15} N(T, A c c)= & p_{00}+p_{10} \cdot T+p_{01} \cdot A c c \\
& +p_{20} \cdot T^{2}+p_{02} \cdot A c c^{2} \\
& +p_{30} \cdot T^{3}+p_{11} \cdot T \cdot A c c \\
& +p_{21} \cdot T^{2} \cdot A c c+p_{12} \cdot T \\
& \cdot A c c^{2}\end{aligned}$} & $p_{02}:$ & $\begin{array}{l}-392.3 \\
(-422.8,-361.8) \\
\end{array}$ & {$\left[\frac{y r^{2} \cdot \% 0}{m^{2}}\right]$} & & \\
\hline & $p_{30}:$ & $\begin{array}{l}1.571 \cdot 10^{-4} \\
(1.465,1.677) \cdot 10^{-4}\end{array}$ & {$\left[\% / K^{3}\right]$} & & \\
\hline & $p_{11}:$ & $\begin{array}{l}19.47 \\
(18.5,20.45) \\
\end{array}$ & {$\left[\frac{y r \cdot \% 0}{m \cdot K}\right]$} & & \\
\hline & $p_{21}:$ & $\begin{array}{l}-0.04268 \\
(-0.04481,-0.04055) \\
\end{array}$ & {$\left[\frac{y r \cdot \% 0}{m \cdot K^{2}}\right]$} & & \\
\hline & $p_{12}:$ & $\begin{array}{l}1.699 \\
(1.571,1.827)\end{array}$ & {$\left[\frac{y r^{2} \cdot \% 0}{m^{2} \cdot K}\right]$} & & \\
\hline
\end{tabular}

Table S01: Two dimensional polynomial surface fit for the static and dynamic model behaviour: coefficients with units, correlationcoefficient $\mathbf{r}^{2}$ and root-mean-squared error RMSE for the polynomial of degree 3 in temperature (T) and degree 2 in accumulation rate (Acc). Note: temperature is in Kelvin. 


\begin{tabular}{ccc}
\hline Scenario: & cop [yr] & s \\
\hline S1 & 1135 & 0.2065 \\
S2 & 1007 & 0.3967 \\
S3 & 1177 & 0.4002 \\
S4 & 1315 & 0.2952 \\
S5 & 1244 & 0.2388 \\
\hline
\end{tabular}

Table S02: Cut off periods and s values for creating the smooth temperature scenarios according to the Monte Carlo approach. 